\title{
Long-term natural history of idiopathic epiretinal membranes with good visual acuity
}

\author{
Kieu-Yen Luu $\mathbb{D}^{1} \cdot$ Tynisha Koenigsaecker $^{1}$ - Amirfarbod Yazdanyar ${ }^{1}$ - Lekha Mukkamala ${ }^{1}$.

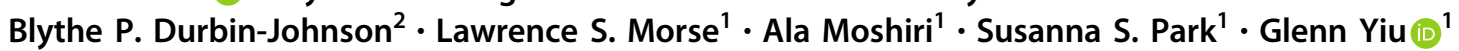

Received: 12 June 2018 / Revised: 8 November 2018 / Accepted: 24 November 2018 / Published online: 19 April 2019

(c) The Royal College of Ophthalmologists 2019

\section{Learning Objectives}

Upon completion of this activity, participants will be able to:

1. Describe long-term progression of iERMs in eyes with good baseline visual acuity, according to a retrospective case series

2. Determine predictors of visual decline in iERMs in eyes with good baseline visual acuity, according to a retrospective case series

3. Explain factors associated with earlier surgical intervention for iERMs in eyes with good baseline visual acuity, according to a retrospective case series

\section{Continuing Medical Education}

In support of improving patient care, this activity has been planned and implemented by Medscape, LLC and Springer Nature. Medscape, LLC is jointly accredited by the Accreditation Council for Continuing Medical Education (ACCME), the Accreditation Council for Pharmacy Education (ACPE), and the American Nurses Credentialing Center (ANCC), to provide continuing education for the healthcare team.

Medscape, LLC designates this Journal-based CME activity for a maximum of 1.00 AMA PRA Category 1 Credit(s). Physicians should claim only the credit commensurate with the extent of their participation in the activity.

All other clinicians completing this activity will be issued a certificate of participation. To participate in this journal CME activity: (1) review the learning objectives and author disclosures; (2) study the education content; (3) take the post-test with a 75\% minimum passing score and complete the evaluation at www.medscape.org/journal/eye; (4) view/print certificate.

\section{Credit hours}

1.0

Release date: 19 April 2019

Expiration date: 19 April 2020

Post-test link: https://medscape.org/eye/posttest909693

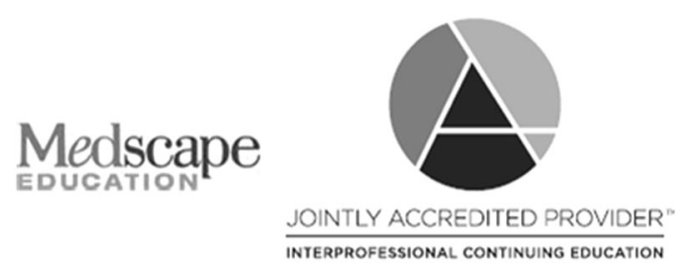

Authors/Editors disclosure information

S.S. has disclosed the following relevant financial relationships: Served as an advisor or consultant for: Allergan, Inc.;

These authors contributed equally: Kieu-Yen Luu, Tynisha Koenigsaecker Bayer AG; Boehringer Ingelheim Pharmaceuticals, Inc.; Heidelberg Pharma GmbH; Novartis Pharmaceuticals

Supplementary information The online version of this article (https:// doi.org/10.1038/s41433-019-0397-z) contains supplementary material, which is available to authorized users. Corporation; Optos; Roche. Served as a speaker or a member of a speakers bureau for: Allergan, Inc.; Bayer AG;

Glenn Yiu

gyiu@ucdavis.edu

1 Department of Ophthalmology \& Vision Sciences, University of California, Davis, Sacramento, CA, USA

2 Department of Public Health Sciences, University of California, Davis, Sacramento, CA, USA 
Novartis Pharmaceuticals Corporation; Optos. Received grants for clinical research from: Allergan, Inc.; Bayer AG; Boehringer Ingelheim Pharmaceuticals, Inc.; Novartis Pharmaceuticals Corporation; Optos. L.S.M. has disclosed the following relevant financial relationships: Served as an advisor or consultant for: Genentech, Inc.; IRIDEX Corporation. Served as a speaker or a member of a speakers bureau for: Genentech, Inc.; IRIDEX Corporation. Received grants for clinical research from: Genentech, Inc. S.S.P. has disclosed the following relevant financial relationships: Received grants for clinical research from: Allergan, Inc.; Roche/Novartis Pharmaceuticals Corporation. G.Y. has disclosed the following relevant financial relationships: Served as an advisor or consultant for: Alimera Sciences; Allergan, Inc.; Carl Zeiss Meditec; IRIDEX Corporation. Received grants for clinical research from: Alcon Laboratories, Inc.; IRIDEX Corporation. K-Y.L., T. K., A.Y., L.M., B.P.D-J., A.M. have disclosed no relevant financial relationships.

\title{
Journal CME author disclosure information
}

Laurie Barclay has disclosed no relevant financial relationships.

\begin{abstract}
Background/objectives To evaluate the long-term progression of idiopathic epiretinal membranes (iERMs) with good baseline visual acuity, and to identify predictors of visual decline.

Design Retrospective case series

Subjects methods We reviewed records of 145 eyes with iERM and best-corrected visual acuity (BCVA) of 20/40 or greater at presentation, including BCVA, lens status, and central macular thickness (CMT) at yearly visits; as well as anatomic biomarkers including vitreomacular adhesion, pseudohole, lamellar hole, intraretinal cysts, disorganization of the inner retinal layers (DRIL), and disruption of outer retinal layers. Linear mixed effects and mixed-effects Cox proportional hazards models were used to identify clinical and anatomic predictors of vision change and time to surgery.

Results At presentation, mean BCVA was $0.17 \pm 0.10 \log$ MAR units (Snellen 20/30) and mean CMT was $353.3 \pm 75.4 \mu \mathrm{m}$. After a median follow-up of 3.7 years (range 1-7 years), BCVA declined slowly at $0.012 \pm 0.003 \operatorname{logMAR}$ units/year, with phakic eyes declining more rapidly than pseudophakic eyes $(0.019 \pm 0.003$ vs. $0.010 \pm 0.004 \operatorname{logMAR}$ units/year). Metamorphopsia, phakic lens status, lamellar hole, and inner nuclear layer cysts were associated with faster visual decline. Cumulative rates of progression to surgery were $2.9,5.6,12.2$, and $21.1 \%$ at years $1-4$. Visual symptoms, metamorphopsia, greater CMT, and disruption of outer retinal layers were associated with greater hazard for surgery.

Conclusion Eyes with iERM and visual acuity $\geq 20 / 40$ experience slow visual decline, with $21 \%$ of eyes requiring surgery after 4 years. Clinical and anatomic predictors of vision loss may be distinct from factors associated with earlier surgical intervention.
\end{abstract}

\section{Introduction}

Epiretinal membranes (ERMs) are a common retinal condition characterized by the growth of fibrocellular tissue on the inner surface of the retina. While ERMs may occur as a consequence of retinal vascular diseases, uveitis, trauma, or retinal tears or detachment [1], most are idiopathic (iERM), with an estimated prevalence of $1.02-28.9 \%$ across different ethnic populations [2]. Although surgical intervention with membrane peeling is indicated for symptomatic patients with significant visual compromise or metamorphopsia, most ERMs with visual acuity greater than 20/40 are monitored until the vision worsens. With advances in microsurgical techniques and improved efficiency of small-gauge vitrectomy systems [3], however, some physicians advocate earlier surgical intervention to maximize visual outcomes [4]. Thus, an understanding of the long-term natural progression of iERM is necessary to provide prognostic guidance for surgical decision-making.
Based on masked grading of fundus photographs, the Blue Mountains Eye Study showed that $9.3 \%$ of early ERMs, termed "cellophane macular reflex," progressed over 5 years to more advanced "preretinal macular fibrosis," which demonstrate visible retinal folds on fundus examination [5]. Although the majority of ERMs remained stable or even regressed in size, $28.6 \%$ of ERMs increased by more than $25 \%$ in area during the 5-year period [5]. However, evaluating ERM progression from fundus photographs is unreliable, and long-term anatomic assessments of iERMs using optical coherence tomography (OCT) have not yet been pursued, due in part to the slow rate of progression of this condition. Also, OCT biomarkers that may predict visual decline or time to surgery have not been extensively explored. In this study, we report the long-term natural history of iERM with no ocular co-morbidities and good visual acuity in patients seen over a 10 -year period, and investigate clinical and OCT-based predictors of visual decline and progression to surgery. 


\section{Methods}

\section{Patient selection}

We reviewed the medical records of 1080 patients diagnosed with an ERM (ICD9 code 362.56 or ICD10 code H35.37x) who were seen at the University of California, Davis Eye Center between 2007 and 2017. This study was approved by the Institutional Review Board of University of California, Davis and was conducted in accordance with the tenets of the Declaration of Helsinki. Eyes with any other ocular comorbidities or history of ocular surgery except uncomplicated cataract extraction were excluded (Supplementary Fig. 1). Eyes with vitreomacular traction (VMT) or full-thickness macular holes at baseline were also excluded. Patients with diabetes mellitus without retinopathy, or glaucoma suspects without visual field loss were not excluded. Only eyes with best-corrected visual acuity (BCVA) of 20/40 or better and at least 1-year of follow-up visits with OCT imaging were analyzed (Supplementary Fig. 1). We recorded baseline demographic and clinical data including age, sex, history of diabetes, history of high myopia, presence of visual symptoms, and presence of metamorphopsia. We also recorded BCVA, lens status (phakic or pseudophakic), and central macular thickness (CMT, average thickness within the $1 \mathrm{~mm}$ diameter circle centered at the fovea) at presentation and yearly follow-up visits. Snellen readings for BCVA were converted to a logarithm of the minimal angle of resolution ( $\log$ MAR) scale for statistical analyses. Eyes that underwent cataract extraction surgery during the study period were classified as phakic at visits prior to surgery, and pseudophakic at visits after the surgery. Data were collected from follow-up visits that are closest to 12month intervals from the initial visit, and where OCT data were also available, until the most recent annual visit, or until membrane peeling surgery was performed. Visits within 1-month of any cataract surgeries were excluded.

\section{Image selection and analysis}

OCT images were obtained using either the Cirrus SD-OCT device (Carl Zeiss Meditec, Dublin, CA) or the Spectralis $\mathrm{OCT}+$ scanning laser ophthalmoscopy (OCT + SLO) device (Heidelberg Engineering, Heidelberg, Germany). Only images obtained longitudinally on the same OCT system, with image registration enabled, were selected for each patient. CMT was automatically determined using the respective OCT software algorithm, and care was taken to exclude images with imaging artifacts such as incorrect foveal centration and/or inaccurate segmentation. For grading of other OCT biomarkers beside CMT, a single horizontal-line B-scan nearest to the foveal center was chosen for analysis. Cirrus images selected for analysis were captured as 5-line "high-definition" raster scans-each horizontal line scan spans $6 \mathrm{~mm}$ with 1024 A-scans per Bscan, and 4 images averaged per scan. Heidelberg OCT images selected for the study were captured as 7-line "high resolution" raster scans spanning $30^{\circ} \times 5^{\circ}$, with $1536 \mathrm{~A}$ scans per B-scan, and Automated Real-time Tracking setting of 25. De-identified OCT images were extracted with the central $3 \mathrm{~mm}$ segment around the fovea labeled using the OCT software caliper tool, based on the diameter of the inner circle of the Early Treatment Diabetic Retinopathy Study grid. Images were separately evaluated by the two masked OCT graders (AY and LM) for image biomarkers within the central $3 \mathrm{~mm}$ region, including the presence or absence of (1) vitreomacular adhesion (VMA), (2) pseudohole, (3) lamellar hole, (4) intraretinal fluid in the inner nuclear layer (INL), (5) intraretinal fluid in the outer plexiform/nuclear layers (OPL/ONL), (6) disorganization of the retinal inner layers (DRIL), (7) disruption of the external limiting membrane (ELM), and (8) disruption of the photoreceptor inner segment/outer segment junction (IS/OS), also known as the ellipsoid zone (EZ) [6]. The presence of VMA was defined as an elevation of perifoveal vitreous cortex from the retinal surface with attachment of the vitreous cortex at the foveal center, as defined by the International Vitreomacular Traction Study (IVTS) group [7]. Pseudohole status was defined as having invaginated or heaped foveal edges, steep macular contour to the central fovea with near-normal central foveal thickness, and no loss of retinal tissue; while lamellar hole status was defined by an irregular foveal contour, defect in the inner fovea, intraretinal splitting (typically between the OPL and ONL), and intact photoreceptor layer, as described by the IVTS classification system and published literature [7-9]. Eyes with intraretinal fluid were graded for fluid location in the INL and/or OPL/ONL, based on the International Nomenclature for OCT panel consensus [6], and the size of the largest cyst (based on horizontal diameter) as small $(\leq 250$ $\mu \mathrm{m})$, medium $(>250$ and $\leq 500 \mu \mathrm{m})$, or large $(>500 \mu \mathrm{m})$ based on previous studies of OCT biomarkers $[10,11]$. The OPL and ONL were not separately analyzed because OCT imaging incorporates Henle's fiber layer, which is histologically part of the OPL, within the hyporeflective ONL layer $[12,13]$. DRIL was defined as the presence of a region on the B-scan where the boundaries between the ganglion cell and inner plexiform layer complex, INL, and OPL could not be separately identified, as described by Sun et al $[10,14,15]$. ELM and IS/OS(EZ) disruption were defined as any discontinuity in the respective hyperreflective bands [16-19]. Any discrepancies in grading were resolved by adjudication by a senior grader (GY). 


\section{Statistical analysis}

Statistical methods were chosen to account for varying lengths of follow-up, as well as the inclusion of both eyes from some subjects. Factors associated with BCVA at baseline were analyzed using linear mixed effects models, using BCVA (logMAR) as the response, a baseline characteristic as the fixed effect, and a random intercept for subject. Baseline factors associated with BCVA over time were analyzed using linear mixed effects models, using BCVA as the response, a baseline factor, time, and their interaction as fixed effects, and nested random intercepts for subject and eye within subject. The coefficient reported for this analysis is the time-baseline factor interaction. Time to surgery was analyzed using mixed-effects Cox proportional hazards models including a baseline factor as the fixed effect and a random intercept for subject. Median follow-up was estimated from a Kaplan-Meier curve using the reverse censoring method. Mean time to surgery was estimated as the restricted mean from a Kaplan-Meier curve, based on a maximum observed surgery time of 7.69 years. Analyses were conducted using R, version 3.4.3 (R Core Team, 2017). Linear mixed effects models were fitted using the $\mathrm{R}$ package nlme, version 3.1-131, and mixed effects Cox models were fitted using the R package coxme, version 2.2-7.

\section{Results}

\section{Baseline characteristics}

We identified 145 eyes of 118 patients who had a diagnosis of iERM with no other ocular co-morbidities, and baseline BCVA of 20/40 or better. The mean age of patients at presentation was $70.6 \pm 8$ years, with a similar proportion of women and men (Supplementary Table 1). Both eyes were affected in 27 patients, with similar proportion of left and right eyes in the series (Supplementary Table 1). Most eyes were asymptomatic $(66 \%)$ at the time of diagnosis, and $42.9 \%$ of those with symptoms also reported the presence of metamorphopsia. Mean baseline BCVA was $0.17 \pm 0.10$ $\log$ MAR units (Snellen equivalent 20/30). No clinical factors including age, sex, diabetes, laterality, symptoms, metamorphopsia, high myopia, or lens status were associated with BCVA at baseline (Supplementary Table 2).

At presentation, mean CMT was $353.3 \pm 75.4 \mu \mathrm{m}$. Based on masked grading of OCT images, VMA was clearly seen in $18 \%$ of gradable cases. Some iERM cases were accompanied by a pseudohole $(6 \%)$ or lamellar hole $(9 \%)$. Intraretinal fluid was seen in 30 eyes $(21 \%)$ with $12 \%$ in the INL and $18 \%$ in the OPL/ONL, and most fluid occurring in small cystoid spaces $(71 \%$ with $<250 \mu \mathrm{m}$ vs. $21 \%$ with $250-500 \mu \mathrm{m}$ and $8 \%$ with $\geq 500 \mu \mathrm{m}$ cysts) (Supplementary Table 1 ). DRIL was noted in $66 \%$ of eyes. ELM disruption occurred in $17 \%$ of cases and IS/OS(EZ) disruptions were noted in $41 \%$. The presence of a pseudohole was the only OCT biomarker associated with better BCVA at presentation $(P=0.024)$. Neither CMT or any other OCT biomarkers were associated with baseline vision (Supplementary Table 2).

\section{Visual anatomic progression}

Median follow-up was 3.7 years (range 1-7 years). While the majority of cases remained stable during the study period, some ERMs progressed rapidly (Figs. 1a-d) while few eyes showed spontaneous improvement (Figs. 1e-h). Overall, visual acuity declined very slowly at a mean rate of $0.012 \pm 0.003 \log$ MAR units per year based on a linear mixed effects model, from BCVA of $0.17 \pm 0.10 \log$ MAR units (Snellen 20/30) at baseline, to a projected BCVA of $0.26 \pm 0.02 \log$ MAR units (Snellen 20/36) at 7 years. Phakic eyes had slightly worse visual acuity at baseline, and also showed greater visual decline than pseudophakic eyes during the study period (Figs. 2a, b), although the difference did not reach statistical significance $(0.019 \pm 0.003$ vs. $0.010 \pm 0.004 \log$ MAR units per year; $P=0.097$ ) based on a linear mixed effects model using time-varying lens status. CMT also increased slowly overall at a mean rate of $2.70 \pm$ $0.74 \mu \mathrm{m}$ per year (Figs. 2c, d), with no significant difference between phakic and pseudophakic eyes $(2.57 \pm 0.92$ vs. $2.99 \pm 1.47 \mu \mathrm{m}$ per year; $P=0.805$ ). Together, these results suggest that anatomic progression of iERMs are similar between phakic and pseudophakic eyes, but that visual decline in phakic eyes may occur more rapidly, likely due to worsening lens opacity.

Factors associated with more rapid visual decline include the presence of metamorphopsia and phakic lens status at presentation, as well as OCT findings of a lamellar hole and INL cysts (Table 1). The presence of IS/OS(EZ) disruption was associated with visual decline on univariate analysis, but was not confirmed in the multivariate model (Table 1). The presence of diabetes mellitus was not associated with the rate of visual decline.

\section{Progression to surgery}

Overall, 21 out of 145 eyes underwent surgical intervention during the study period. Cumulative rates of progression to surgery were $2.9 \%$ at year $1,5.6 \%$ at year $2,12.2 \%$ at year 3 , and $21.1 \%$ at year 4 based on Kaplan-Meier estimates (Fig. 3a). The mean time to surgery was 6.5 years, and mean visual acuity at time of surgery was $\log$ MAR $0.40 \pm 0.15$ (Snellen 20/50) (Table 1). At any time, a higher proportion of eyes that were pseudophakic at baseline underwent ERM surgery than eyes that were phakic at baseline (Fig. 3b). As expected, the presence of symptoms $(P=0.008)$ and 

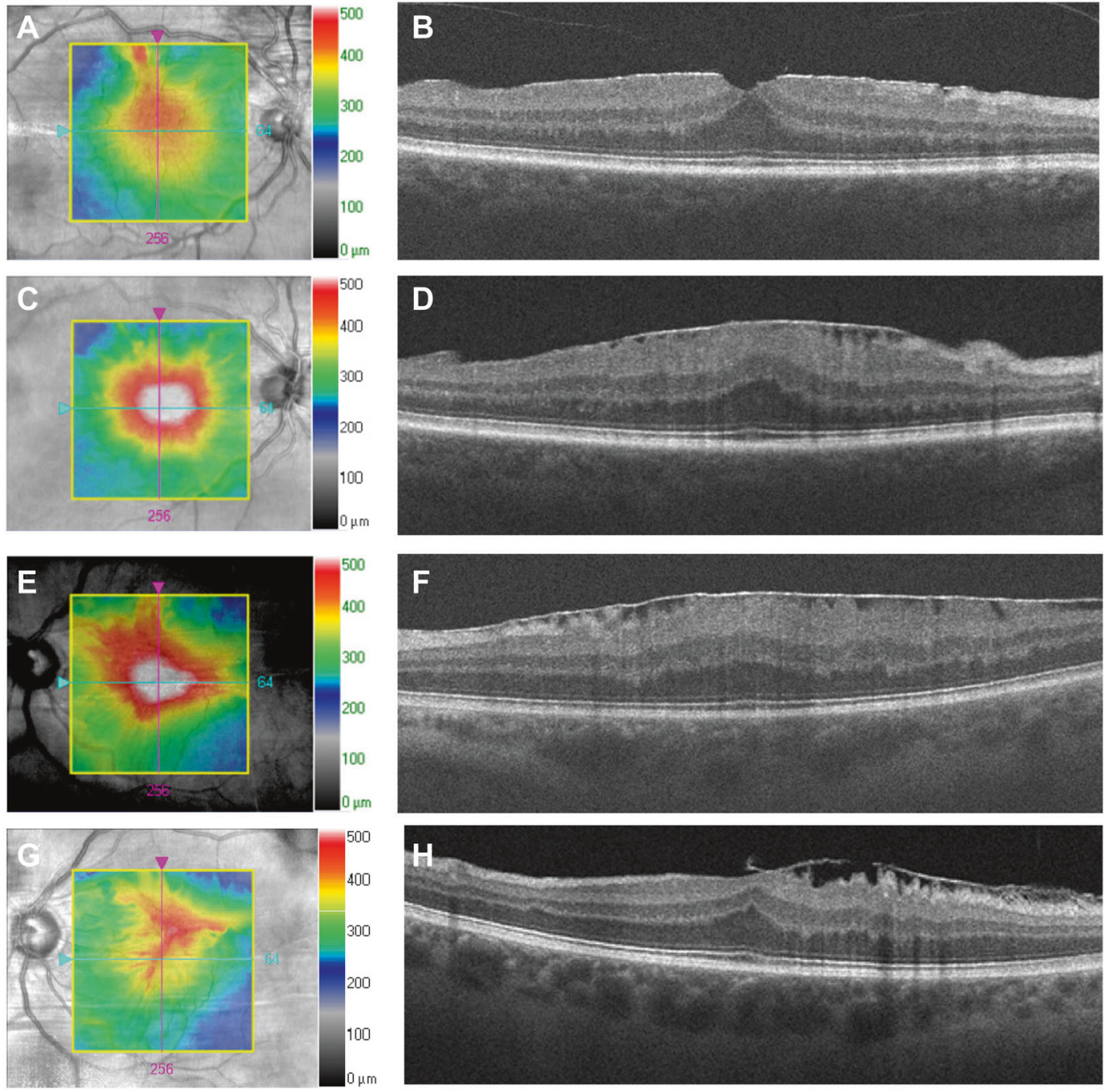

Fig. 1 Different patterns of long-term idiopathic ERM progression. Infrared images with topographic overlay $(\mathbf{a}, \mathbf{c}, \mathbf{e}, \mathbf{g})$ and OCT images $(\mathbf{b}, \mathbf{d}, \mathbf{f}, \mathbf{h})$ show two patients with different patterns of ERM progression. Patient RT (a-d) had a mild ERM and good visual acuity 20/ 20 at presentation $(\mathbf{a}-\mathbf{b})$, but progressed after 3 years with diffuse

metamorphopsia $(P=0.050)$ at baseline were associated with greater hazard for surgical intervention. The presence of diabetes did not impact time to surgery. OCT predictors for surgical intervention include higher baseline CMT $(P=$ $0.009)$, presence of ELM disruption $(P=0.001)$, and presence of IS/OS(EZ) disruption $(P=0.009)$ (Table 2). Comparison of baseline clinical and OCT characteristics between eyes that underwent surgery vs. those that did not undergo surgery during the follow-up period help illustrate the impact of these factors (Supplementary Table 1). In eyes that underwent surgery $(n=21)$, median preoperative visual acuity was $0.398 \log$ MAR (20/50), and improved significantly to $0.301 \operatorname{logMAR}(20 / 40)$ after surgery $(P<$ 0.001 ) after exclusion of 4 surgical patients who had postoperative complications (cystoid macular edema, corneal retinal thickening, inner retinal striae, and visual decline to 20/50 (c-d). Patient AM (e-h) had an idiopathic ERM with visual acuity of 20/30 at presentation (e-f), which spontaneously contracted with some restoration of foveal contour and visual improvement to 20/20 after 5 years $(\mathbf{g}-\mathbf{h})$

edema, and retinal edema). The only factors associated with poor visual recovery after surgery was the presence of OPL/ ONL cysts on baseline OCT (Supplementary Table 3).

\section{Discussion}

The timing for surgical intervention for iERM removal has been a topic of controversy, with some potential benefits for early removal, but also a lack of negative consequences to waiting for a limited time [20,21]. Although some studies suggest superior visual outcomes after iERM removal while visual acuity is better than $20 / 40[4,22]$, many clinicians monitor iERMs until the patient becomes symptomatic or visual acuity declines to $20 / 40$ or below. Thus, clinical and 
A

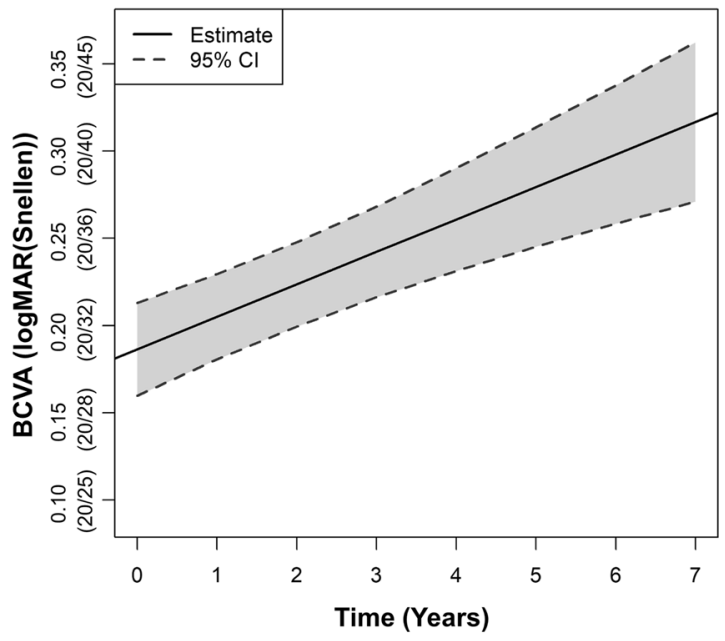

C

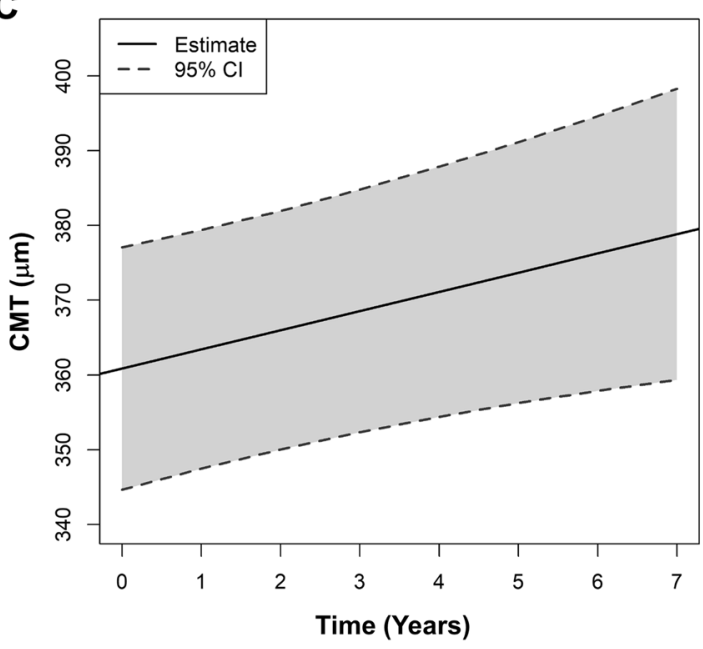

Fig. 2 Visual and anatomic progression of idiopathic ERM by lens status. Plots show estimates from linear mixed effects model for BCVA (a-b) and CMT (c-d) by time-varying lens status for phakic $(\mathbf{a}, \mathbf{c})$ and pseudophakic $(\mathbf{b}, \mathbf{d})$ eyes. The model fit for each plot is

OCT characteristics that predict rate of visual decline or time to surgery may better inform decision and timing for surgical intervention.

In this study, we found that patients with iERM and good visual acuity maintain excellent vision long-term at up to 7 years of follow-up, with $21 \%$ undergoing surgery from years 4 to 7 . Our results are consistent with smaller caseseries of iERMs that demonstrate relative stability in BCVA and CMT over 24 months [23], and a $22 \%$ progression rate to membrane peeling at 6.5 years [24]. Based on our cohort, linear mixed effects modeling predicts less than one Snellen acuity line of vision loss over 7 years, although there is some heterogeneity among individual cases with some iERM progressing more rapidly and others improving spontaneously (Fig. 1). While these cases are uncommon in our cohort, a more focused case series of these rapidly
B

Pseudophakic
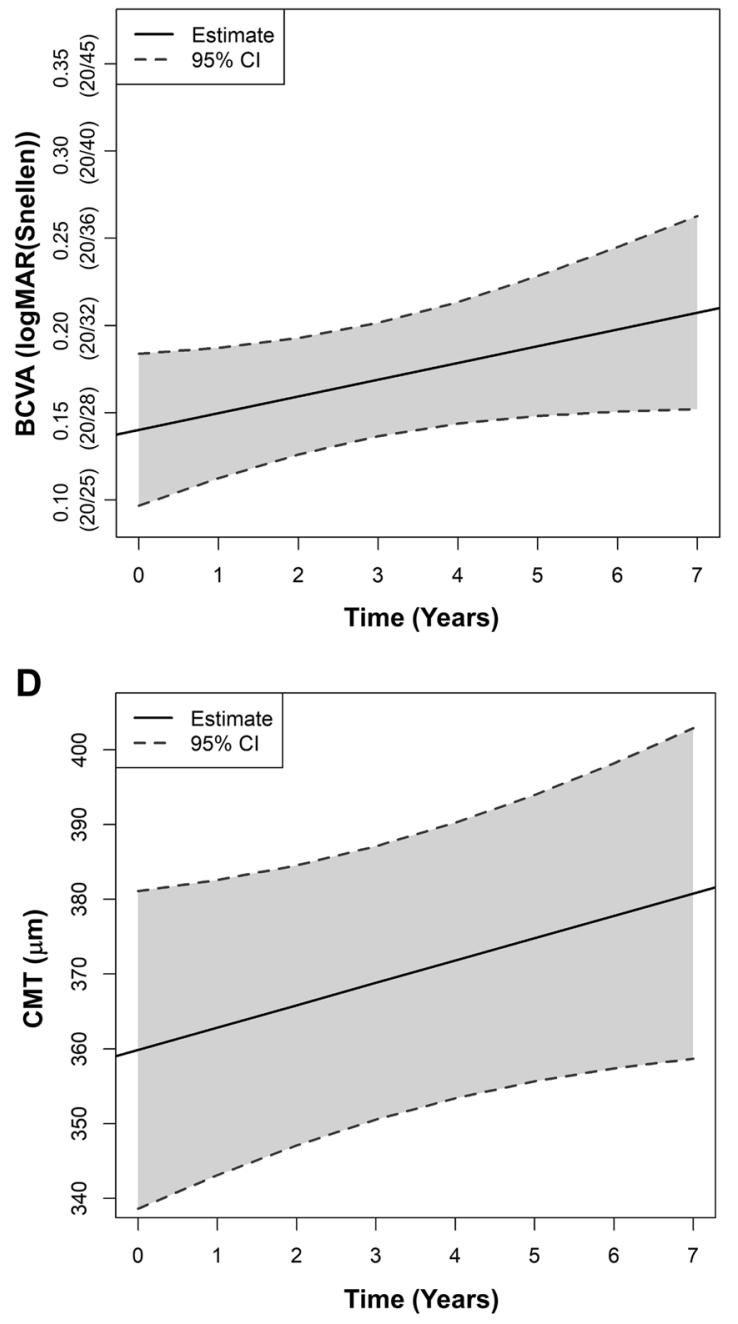

shown as a solid line, and the $95 \% \mathrm{CI}$ is shown as dashed lines. Abbreviations: $B C V A$ best-corrected visual acuity, $C I$ confidence intervals, $C M T$ central macular thickness, ERM epiretinal membranes

progressing or spontaneously resolving iERMs may be of interest for clinical practice. By using a linear model with time-varying lens status in our study, we were also able to estimate the vision loss attributed solely to iERM progression independent of lens status, with phakic eyes experiencing roughly doubled the rate of visual decline than pseudophakic eyes $(0.019 \pm 0.003$ vs. $0.010 \pm 0.004 \log$ MAR units per year). Since cataract severity was not graded in this retrospective analysis, future studies that employ more precise cataract grading or other functional measures such as microperimetry or metamorphopsia may provide a more robust understanding of iERM progression. Interestingly, although phakic eyes experience more visual decline than pseudophakic eyes with iERM, they are also less likely to undergo membrane peeling surgery at any time (Fig. 3b). While the decision for surgery was not standardized and 
Table 1 Baseline factors associated with visual acuity change

\begin{tabular}{|c|c|c|c|}
\hline Univariate models & Coefficient $(95 \% \mathrm{CI})$ & SE & $P$-value ${ }^{\mathrm{a}}$ \\
\hline \multicolumn{4}{|l|}{ Clinical characteristics } \\
\hline Baseline age & $3 \mathrm{e}-04(-0.00038,0.00098)$ & 0.00035 & 0.387 \\
\hline Sex (male) & $-0.0048(-0.015,0.0053)$ & 0.0051 & 0.352 \\
\hline Diabetes mellitus & $0.0056(-0.0065,0.018)$ & 0.0062 & 0.362 \\
\hline Laterality (right) & $-0.009(-0.019,0.0011)$ & 0.0051 & 0.081 \\
\hline Symptoms & $0.0012(-0.011,0.013)$ & 0.0062 & 0.844 \\
\hline Metamorphopsia & $0.026(0.0047,0.047)$ & 0.011 & 0.017 \\
\hline High myopia $>6$ diopters & $-0.008(-0.024,0.0081)$ & 0.0082 & 0.330 \\
\hline Baseline lens status (phakic) & $-0.014(-0.027,-0.0015)$ & 0.0064 & 0.028 \\
\hline \multicolumn{4}{|l|}{ OCT characteristics } \\
\hline Baseline CMT & $4.2 \mathrm{e}-05(-3.1 \mathrm{e}-05,0.00011)$ & $3.7 \mathrm{e}-05$ & 0.256 \\
\hline VMA & $0.032(-0.041,0.1)$ & 0.036 & 0.385 \\
\hline Pseudohole & $-0.005(-0.023,0.013)$ & 0.0094 & 0.592 \\
\hline Lamellar hole & $0.021(0.0074,0.035)$ & 0.0071 & 0.003 \\
\hline INL cysts & $0.024(0.011,0.038)$ & 0.007 & 0.001 \\
\hline OPL/ONL cysts & $-0.0047(-0.018,0.0084)$ & 0.0067 & 0.479 \\
\hline Size of largest cyst (large vs. small) & $-0.014(-0.046,0.018)$ & 0.016 & 0.380 \\
\hline Size of largest cyst (medium vs. small) & $-0.033(-0.082,0.017)$ & 0.025 & 0.194 \\
\hline DRIL disruption & $0.0032(-0.0077,0.014)$ & 0.0055 & 0.568 \\
\hline ELM disruption & $0.0058(-0.0098,0.021)$ & 0.0079 & 0.466 \\
\hline IS/OS(EZ) disruption & $0.011(0.00067,0.021)$ & 0.0052 & 0.037 \\
\hline Multivariate model & Coefficient $(95 \% \mathrm{CI})$ & SE & $P$-value ${ }^{\mathrm{a}}$ \\
\hline \multicolumn{4}{|l|}{ Clinical characteristics } \\
\hline Metamorphopsia & $0.031(0.0092,0.053)$ & 0.011 & 0.005 \\
\hline Baseline lens status (phakic) & $-0.015(-0.028,-0.0026)$ & 0.0064 & 0.018 \\
\hline \multicolumn{4}{|l|}{ OCT characteristics } \\
\hline Lamellar hole & $0.021(0.0067,0.034)$ & 0.0071 & 0.004 \\
\hline INL cysts & $0.02(0.0058,0.033)$ & 0.007 & 0.005 \\
\hline IS/OS(EZ) disruption & $0.0085(-0.0014,0.019)$ & 0.0051 & 0.093 \\
\hline
\end{tabular}

BCVA best-corrected visual acuity, $C I$ confidence interval, CMT central macular thickness, DRIL disorganization of the retinal inner layers, $E L M$ external limiting membrane, $E Z$ ellipsoid zone, $I S$ inner segment, LogMAR logarithm of the minimum angle of resolution, INL inner nuclear layer, OCT optical coherence tomography, $O N L$ outer nuclear layer, $O P L$ outer plexiform layer, $O S$ outer segment, $S E$ standard error $V M A$ vitreomacular adhesion

${ }^{a}$ From linear mixed effects model. Coefficient is difference between groups in rate of change of logMAR over time for categorical covariate, and change in rate of change over time for unit increase in continuous covariate

likely multifactorial in this retrospective case series, we suspect that clinicians who care for patients with both cataracts and iERM are more likely to offer cataract extraction rather than membrane surgery as an initial option.

In our study, we identified INL fluid and lamellar holes as anatomic predictors of visual decline in patients with iERM. Prior studies have demonstrated the detrimental impact of intraretinal fluid on visual function in eyes with iERM [25, 26], as well as other retinal conditions such as age-related macular degeneration [27] and diabetic macular edema [28]. Lamellar holes with an ERM have also been associated with worse vision than those without an ERM, and visual outcomes after
ERM peeling surgery are worse in the presence of a lamellar hole [29]. Unlike pseudoholes, which primarily involves tangential traction along the vitreomacular interface, lamellar holes are characterized by dehiscence of the inner foveal retina from the outer retina [30], which may be associated with defects in Müller cell processes that span the internal and external limiting membranes, and may thus more severely impact visual function [31]. We did not find in our iERM cohort an association between vision change and other OCT characteristics such as DRIL or IS/OS(EZ) disruption which are predictors of visual acuity in other retinal conditions [10, 14-19, 32]. While this disparity may reflect the different pathophysiology of 


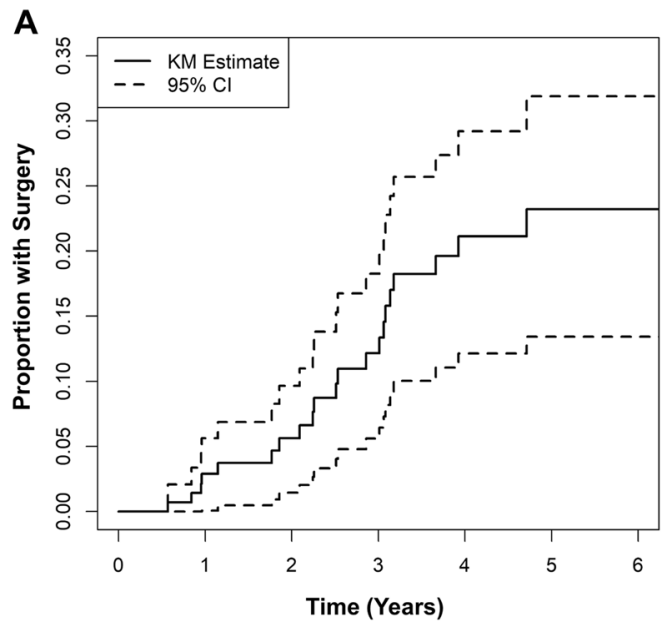

Fig. 3 Progression to surgery for idiopathic ERM. Kaplan-Meier plot of time to surgery is shown for all eyes (a), and eyes categorized by baseline lens status (b). The estimate for all eyes is shown as a solid line, and the $95 \% \mathrm{CI}$ is shown as dashed lines in (a). The estimate for
B

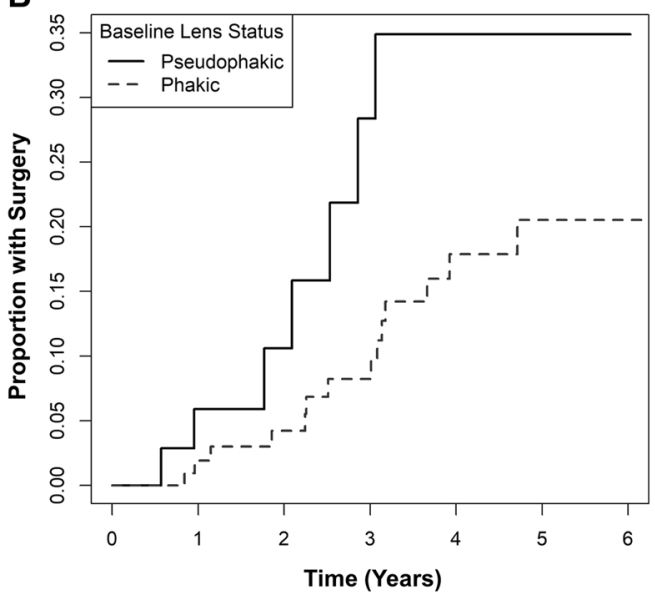

pseudophakic eyes is shown as a solid line, and the estimate for phakic eyes is shown as a dashed line in (b). Abbreviations: $C I$ confidence interval, ERM epiretinal membrane, $K M$ Kaplan-Meier
Table 2 Factors associated with time to surgery

\begin{tabular}{llll}
\hline & Hazard Ratio $(95 \%$ CI $)$ & Coefficient \pm SE & $P$-value \\
\hline Clinical characteristics & & & \\
Baseline age & $0.98(0.92,1.04)$ & $-0.02 \pm 0.031$ & 0.530 \\
Sex (male) & $1.95(0.75,5.09)$ & $0.67 \pm 0.488$ & 0.170 \\
Diabetes mellitus & $0.63(0.17,2.34)$ & $-0.465 \pm 0.672$ & 0.489 \\
Laterality (right) & $1.03(0.42,2.56)$ & $0.034 \pm 0.463$ & 0.941 \\
Symptoms & $3.4(1.37,8.43)$ & $1.224 \pm 0.463$ & 0.008 \\
Metamorphopsia & $2.78(1,7.72)$ & $1.022 \pm 0.521$ & 0.050 \\
High myopia $>6$ diopters & n.e. & n.e. & n.e. \\
Baseline lens status (phakic) & $0.4(0.14,1.11)$ & $-0.921 \pm 0.525$ & 0.079 \\
OCT characteristics & & & \\
Baseline CMT & $1.008(1.002,1.014)$ & $0.008 \pm 0.003$ & 0.009 \\
VMA & $0.42(0.04,4.32)$ & $-0.859 \pm 1.184$ & 0.468 \\
Pseudohole & $0.55(0.07,4.65)$ & $-0.594 \pm 1.087$ & 0.585 \\
Lamellar hole & n.e. & n.e. & n.e. \\
INL cysts & $1.24(0.34,4.6)$ & $0.217 \pm 0.668$ & 0.746 \\
OPL/ONL cysts & $0.27(0.03,2.05)$ & $-1.327 \pm 1.044$ & 0.204 \\
Size of largest cyst (large vs. small) & n.e. & n.e. & n.e. \\
Size of largest cyst (medium vs. small) & n.e. & n.e. & n.e. \\
DRIL disruption & $1.35(0.48,3.81)$ & $0.299 \pm 0.53$ & 0.573 \\
ELM disruption & $7.86(2.35,26.32)$ & $2.062 \pm 0.617$ & 0.001 \\
IS/OS(EZ) disruption & $3.85(1.39,10.62)$ & $1.347 \pm 0.518$ & 0.009 \\
\hline BCVA bestcotrcted visua & a & & \\
\hline
\end{tabular}

BCVA best-corrected visual acuity, $C I$ confidence interval, CMT central macular thickness, DRIL disorganization of the retinal inner layers, ELM external limiting membrane, EZ ellipsoid zone, IS inner segment, LogMAR logarithm of the minimum angle of resolution, INL inner nuclear layer, n.e. not estimable due to no events in one category, $O C T$ optical coherence tomography, $O N L$ outer nuclear layer, $O P L$ outer plexiform layer, $O S$ outer segment, $S E$ standard error, $V M A$ vitreomacular adhesion

${ }^{a}$ From mixed effects Cox proportional hazards model, using Wald test. Coefficient is log hazard ratio
iERMs, we cannot exclude the possibility that potential associations may be muted by focusing only on iERMs with good visual acuity and slow progression.
With respect to surgical intervention, patients who reported visual symptoms and/or metamorphopsia at presentation were more likely to undergo surgery for ERM 
removal, which is not surprising. Interestingly, OCT predictors for surgical intervention were different from those that predict visual decline. In our series, eyes with greater CMT and disruption of outer retinal layers (ELM and IS/OS (EZ)) were more likely to undergo surgery, while those with a lamellar hole and INL fluid exhibit more rapid visual decline. These findings are interesting, as they suggest that OCT characteristics that impact a patient's visual perception or the clinician's surgical decision-making may be different from biomarkers of disease progression or vision loss. iERMs that exhibit greater retinal thickening on OCT, for example, may be more likely to be operated upon, even if CMT is not independently predictive of visual decline. Nevertheless, such conclusions cannot be easily drawn given the retrospective nature of this study, and the absence of strict criteria for surgical intervention afforded by a prospective analysis. Future studies that rely on unbiased approaches, such as using machine learning algorithms to analyze OCTs, may provide better predictive value for iERM progression and the associated vision loss, and help clinicians better inform patients regarding the decision for surgical intervention.

\section{Summary}

\section{What was known before}

- Epiretinal membranes (ERM) can lead to progressive vision loss and require membrane peeling surgery, but patients with early ERMs and good visual acuity are often monitored. The long-term natural history of ERMs and factors that predict visual decline are unclear, and may help clinicians decide on the need for surgical intervention.

\section{What this study adds}

- Eyes with idiopathic epiretinal membranes and good visual acuity greater than 20/40 undergo gradual but mild vision loss over a median follow-up of 4 years, with $21 \%$ of eyes requiring surgery in this retrospective series. Clinical and anatomic predictors of vision loss may be distinct from factors associated with earlier surgical intervention.

Acknowledgements KYL: none, Tynisha Koenigsaecker: none, AY: none, LM: none, BDJ: NIH UL1 TR001860 (National Center for Advancing Translational Sciences), LSM: none, AM: NIH K08 EY027463, Research to Prevent Blindness, International Retinal Research Foundation, SSP: none, GY: NIH K08 EY026101, E Matilda Ziegler Foundation for the Blind, ARVO Foundation, Alcon Research
Institute, California National Primate Research Center, CITRIS/ Banatao Institute

\section{Compliance with ethical standards}

Conflict of interest Lawrence S. Morse: consultancy fees from Genentech. Glenn Yiu: grants from Alcon, Genentech, Iridex; and consultancy fees from Alimera, Allergan, Carl Zeiss Meditec, Iridex, and Southern California Desert Retina. The remaining authors declare that they have no conflict of interest.

Publisher's note: Springer Nature remains neutral with regard to jurisdictional claims in published maps and institutional affiliations.

\section{References}

1. Hejsek L, Stepanov A, Dohnalova A, Rehakova T, Jiraskova N. The natural evolution of idiophatic epimacular membrane. Biomed Pap Med Fac Univ Palacky Olomouc Czech Repub. 2017;161:100-6.

2. Bu SC, Kuijer R, Li XR, Hooymans JM, Los LI. Idiopathic epiretinal membrane. Retina. 2014;34:2317-35.

3. Konstantinidis L, Berguiga M, Beknazar E, Wolfensberger TJ. Anatomic and functional outcome after 23-gauge vitrectomy, peeling, and intravitreal triamcinolone for idiopathic macular epiretinal membrane. Retina. 2009;29:1119-27.

4. Moisseiev E, Kinori M, Moroz I, Priel E, Moisseiev J. 25-Gauge vitrectomy with epiretinal membrane and internal limiting membrane peeling in eyes with very good visual acuity. Curr Eye Res. 2016;41:1387-92.

5. Fraser-Bell S, Guzowski M, Rochtchina E, Wang JJ, Mitchell P. Five-year cumulative incidence and progression of epiretinal membranes: the Blue Mountains Eye Study. Ophthalmology. 2003;110:34-40.

6. Staurenghi G, Sadda S, Chakravarthy U, Spaide RF, International Nomenclature for Optical Coherence Tomography P. Proposed lexicon for anatomic landmarks in normal posterior segment spectral-domain optical coherence tomography: the IN*OCT consensus. Ophthalmology. 2014;121:1572-8.

7. Duker JS, Kaiser PK, Binder S, de Smet MD, Gaudric A, Reichel E, et al. The International Vitreomacular Traction Study Group classification of vitreomacular adhesion, traction, and macular hole. Ophthalmology. 2013;120:2611-9.

8. Haouchine B, Massin P, Tadayoni R, Erginay A, Gaudric A. Diagnosis of macular pseudoholes and lamellar macular holes by optical coherence tomography. Am J Ophthalmol. 2004;138:732-9.

9. Hirano M, Morizane Y, Kimura S, Hosokawa M, Shiode Y, Doi S, et al. Assessment of lamellar macular hole and macular pseudohole with a combination of en face and radial B-scan optical coherence tomography imaging. Am J Ophthalmol. 2018;188:29-40.

10. Sun JK, Lin MM, Lammer J, Prager S, Sarangi R, Silva PS, et al. Disorganization of the retinal inner layers as a predictor of visual acuity in eyes with center-involved diabetic macular edema. JAMA Ophthalmol. 2014;132:1309-16.

11. Sun JK, Radwan SH, Soliman AZ, Lammer J, Lin MM, Prager $\mathrm{SG}$, et al. Neural retinal disorganization as a robust marker of visual acuity in current and resolved diabetic macular edema. Diabetes. 2015;64:2560-70.

12. Yiu G, Wang Z, Munevar C, Tieu E, Shibata B, Wong B, et al. Comparison of chorioretinal layers in rhesus macaques using spectral-domain optical coherence tomography and highresolution histological sections. Exp Eye Res. 2018;168:69-76.

13. Lujan BJ, Roorda A, Knighton RW, Carroll J. Revealing Henle's fiber layer using spectral domain optical coherence tomography. Invest Ophthalmol Vis Sci. 2011;52:1486-92. 
14. Das R, Spence G, Hogg RE, Stevenson M, Chakravarthy U. Disorganization of inner retina and outer retinal morphology in diabetic macular edema. JAMA Ophthalmol. 2018;136:202-8.

15. Grewal DS, O’Sullivan ML, Kron M, Jaffe GJ. Association of disorganization of retinal inner layers with visual acuity in eyes with uveitic cystoid macular edema. Am J Ophthalmol. 2017;177:116-25.

16. Theodossiadis PG, Theodossiadis GP, Charonis A, Emfietzoglou I, Grigoropoulos VG, Liarakos VS. The photoreceptor layer as a prognostic factor for visual acuity in the secondary epiretinal membrane after retinal detachment surgery: imaging analysis by spectral-domain optical coherence tomography. Am J Ophthalmol. 2011;151:973-80.

17. Uji A, Murakami T, Nishijima K, Akagi T, Horii T, Arakawa N, et al. Association between hyperreflective foci in the outer retina, status of photoreceptor layer, and visual acuity in diabetic macular edema. Am J Ophthalmol. 2012;153:710-7, 7 e1.

18. Chen X, Zhang L, Sohn EH, Lee K, Niemeijer M, Chen J, et al. Quantification of external limiting membrane disruption caused by diabetic macular edema from SD-OCT. Invest Ophthalmol Vis Sci. 2012;53:8042-8.

19. Coscas F, Coscas G, Lupidi M, Dirani A, Srour M, Semoun O, et al. Restoration of outer retinal layers after aflibercept therapy in exudative AMD: prognostic value. Invest Ophthalmol Vis Sci. 2015;56:4129-34.

20. Rahman R, Stephenson J. Early surgery for epiretinal membrane preserves more vision for patients. Eye. 2014;28:410-4.

21. Kofod M, Christensen UC, la Cour M. Deferral of surgery for epiretinal membranes: Is it safe? Results of a randomised controlled trial. Br J Ophthalmol. 2016;100:688-92.

22. Par GPJ. Should epiretinal membranes be removed before vision drops below 20/40? 35th annual meeting of the American Society of Retina Specialists. Boston, MA; 2017.

23. Byon IS, Pak GY, Kwon HJ, Kim KH, Park SW, Lee JE. Natural history of idiopathic epiretinal membrane in eyes with good vision assessed by spectral-domain optical coherence tomography. Ophthalmologica. 2015;234:91-100.

24. Xuejing Chen CS, Jeffery Heier. Progression to Surgery for Epiretinal Membranes with Good Vision. ARVO; May 10, 2017; Harvard: Ophthalmic Consultants of Boston, Tufts Medical Center; 2017.

25. Michalewski J, Michalewska Z, Cisiecki S, Nawrocki J. Morphologically functional correlations of macular pathology connected with epiretinal membrane formation in spectral optical coherence tomography (SOCT). Graefes Arch Clin Exp Ophthalmol. 2007;245:1623-31.

26. Frisina R, Pinackatt SJ, Sartore M, Monfardini A, Baldi A, Cesana $\mathrm{BM}$, et al. Cystoid macular edema after pars plana vitrectomy for idiopathic epiretinal membrane. Graefes Arch Clin Exp Ophthalmol. 2015;253:47-56.

27. Jaffe GJ, Martin DF, Toth CA, Daniel E, Maguire MG, Ying GS, et al. Macular morphology and visual acuity in the comparison of age-related macular degeneration treatments trials. Ophthalmology. 2013;120:1860-70.

28. Otani T, Kishi S, Maruyama Y. Patterns of diabetic macular edema with optical coherence tomography. Am J Ophthalmol. 1999;127:688-93.

29. Sun JP, Chen SN, Chuang CC, Lin CW, Lin CJ, Huang JY, et al. Surgical treatment of lamellar macular hole secondary to epiretinal membrane. Graefe's Arch Clin Exp Ophthalmol. 2013;251:2681-8.

30. Takahashi H, Kishi S. Tomographic features of a lamellar macular hole formation and a lamellar hole that progressed to a fullthickness macular hole. Am J Ophthalmol. 2000;130:677-9.

31. Spaide RF. Closure of an outer lamellar macular hole by vitrectomy: hypothesis for one mechanism of macular hole formation. Retina. 2000;20:587-90.

32. Pilli S, Lim P, Zawadzki RJ, Choi SS, Werner JS, Park SS. Fourier-domain optical coherence tomography of eyes with idiopathic epiretinal membrane: correlation between macular morphology and visual function. Eye. 2011;25:775-83. 